Original
Article

\title{
Value of Inferior Meatal Window with Mucosal Flap as an Adjuvant to Middle Meatal Antrostomy in Managing Maxillary Sinus Lesions: A Randomized Controlled Trial
}

\author{
Ahmed Amin Nassar, Ahmed ElBatawi, Khaled Azooz, Khaled Harhash, Mohamed \\ Sabaa
}

Department of Otolaryngology, Faculty of Medicine, Cairo University, Cairo, Egypt.

\begin{abstract}
Background: Several approaches for the maxillary sinus were described. Currently, endoscopic Middle Meatal Antrostomy (MMA) is the gold standard for managing maxillary sinus lesions. Unfortunately, there are some limitations especially in hidden areas. This research elucidates the advantages and disadvantages of adding IMF to the MMA for managing maxillary sinus lesions.

Patients and Methods: This study was a randomized controlled trial, conducted on sixty patients, divided into 2 groups: Group (A) included 30 patients underwent IMF after MMA, while group (B) involved 30 patients subjected to MMA only for managing the maxillary sinus. Patients were assessed for the accessibility of the antero-inferior area of the maxillary sinus, residual lesion after performing IMF and evidence of recurrence.

Results: The antero-inferior area accessibility in group (A) was difficult in 10 patients (33\%) compared to 20 patients $(66 \%)$ of group (B) $(P=0.004)$. Residual was detected after performing the IMF in 12 patients $(40 \%)$. Postoperative 1 year Lund Mackay score comparison between both groups was statistically insignificant $(P=0.6)$. Recurrence was noted in 4 cases of group (A) and 2 cases of group $(\mathrm{B}),(P=0.7)$.

Conclusion: Inferior meatal window with mucosal flap is helpful approach to maxillary sinus hidden areas when added to standard MMA. It is best suited for complete removal of small hidden lesions (e.g. foreign bodies, fungal ball and odontogenic cysts) which could be hardly handled through MMA.
\end{abstract}

Key Words: Inferior meatal window, maxillary sinus, MMA.

Received: 8 October 2019, Accepted: 27 April 2020

Corresponding Author: Ahmed Amin Nassar; MD, Department of Otolaryngology, Faculty of Medicine, Cairo University, Cairo, Egypt, Tel.: 201001960709, E-mail: ahmedaminnassar1@kasralainy.edu.eg

ISSN: 2090-0740, November 2020 Vol.21, No.3

\section{INTRODUCTION}

Several approaches for the maxillary sinus have been described $^{[1]}$. The middle meatal antrostomy (MMA) was first described by Siebenmann $(1899)^{[2]}$. A new era in sinus surgery began in the early 1970 s with the development of different angled endoscopes ${ }^{[3]}$.

Endoscopic MMA was introduced by Kennedy and colleagues as a better surgical approach to the maxillary sinus in the mid-1980s $\mathrm{s}^{[4]}$. However, there are some limitations; even after a generous MMA, using angled endoscopes and instruments, challenges arise in the areas of the alveolar recess, the anterior and medial walls ${ }^{[5]}$.

Since the introduction of Functional endoscopic surgery, inferior meatal antrostomy has been receded. Despite fears of mucociliary recirculation; several benefits were obtained: Drainage was facilitated, removal of pathologies (as mycetomas, antrochoanal polyps and allergic fungal disease) and better visualization of the floor and anterior wall of the maxillary sinus ${ }^{[6]}$.
In 2015, Karpischenko ${ }^{[7]}$ proposed the inferior meatus osteo-mucosal posteriorly based flap approach (IMF) for which it is claimed to be effective with less bleeding and postoperative complains compared to other methods.

\section{PATIENTS AND METHODS:}

This study was a randomized controlled trial, with two parallel groups, conducted on sixty patients with maxillary sinus pathology, from March 2016 to August 2017, in one university hospital and approved by the institutional review board. Patients were randomized using sealed envelopes (1:1 allocation) into two groups: Group (A) included thirty patients underwent IMF approach after conventional MMA, while group (B) involved thirty patients subjected to conventional MMA only for managing the maxillary sinus.

Patients over 8 year-old with various forms of maxillary sinus pathology (extending to the antero- 
inferior compartment) were included e.g. allergic polyps, antrochoanal polyps and dental cysts. However, patients with maxillary sinus hypoplasia, lesions not involving antero-inferior area and revision cases were excluded.

Preoperatively, patients were subjected to full history taking, physical and endoscopic examination. Radiologic assessment was done using Lund-Mackay computed tomography (CT) scoring for maxillary sinus and ostiomeatal complex (OMC): $0=$ no opacity, $1=$ partial opacity or $2=$ complete opacity. Scoring was performed by different surgeon, who was blinded to the patients' group.

Intra-operatively; accessibility to the antero-inferior wall of maxillary sinus was assessed in both groups and detection of residual was noted in cases subjected to IMF.

Postoperatively, assessment was done endoscopically (at 2 and 8 weeks) for healing of the flap (group A), radiological assessment (at 1 year follow up) was scored similar to the preoperative one by the same surgeon who scored before. Recurrence of the disease was noted endoscopically and/or from the CT scans after 1 year.

All surgeries for both groups were performed by the same surgeon.

\section{Surgical technique:}

Under general anesthesia, starting with $0^{\circ}$ endoscope, decongestion of the nasal mucosa was achieved by placement of nasal pledgets that contain oxymetazoline $0.05 \%$ in the inferior meatus (group A) and the middle meatus. Maxillary sinus lesions were dealt with using standard MMA with different angled endoscopes $\left(0^{\circ}, 30^{\circ}, 45^{\circ}\right.$ and $\left.70^{\circ}\right)$ and instruments.

Additionally; in group A, with the aid of $0^{\circ}$ and $30^{\circ}$ endoscope, the inferior turbinate and the incision site in the inferior meatus were infiltrated with 1:100000 epinephrine solutions, a Freer elevator was used to up fracture the inferior turbinate for adequate visualization, Hasner's valve was indentified $(15 \mathrm{~mm}$ above the nasal floor and 4-6 $\mathrm{mm}$ posterior to the head of the inferior turbinate), monopolar diathermy or no. 15 blade scalpel was used to make the incision below and anterior to the Hasner's valve, mucoperiosteal flap was elevated with Freer elevator, the inferior portion of the medial maxillary sinus wall was opened using the same instrument at the level of mucosal incision and Heymann turbinectomy scissors were used to create a posteriorly based osteo-mucosal flap with a variable length according to the need. After assessment and dealing with residual lesions, osteo-mucosal flap was returned back to close gap of temporary approach and inferior turbinate was lateralized (Figure 1).
Postoperatively; patients had nasal packs for 48 hours. Amoxicillin-Clavulanate (1gm/12 hours for 7days), Paracetamol $(500 \mathrm{mg} / 8$ hours for 1 week), Saline nasal irrigation and Steroid nasal sprays (in indicated cases) were prescribed.

\section{STATISTICAL ANALYSIS:}

Data were coded and entered using the statistical package SPSS version 25. Data was summarized using mean and standard deviation for quantitative variables and frequencies (number of cases) and relative frequencies (percentages) for categorical variables. Comparisons between groups were done using unpaired t test. For comparing categorical data, Chi square $\left(\chi^{2}\right)$ test was performed. Exact test was used instead when the expected frequency is less than 5. P-values less than 0.05 were considered as statistically significant.

\section{RESULTS:}

In this study, ages ranged from 9 to 61 with a mean of $29 \pm 12$ SD years in group (A) and $31 \pm 11$ SD years in group (B) which was statistically insignificant $(P=0.6)$.

Regarding sex distribution, group (A) included 17 males and 13 females while group (B) had 15 males and 15 females with statistically insignificant $P$ value $(0.6)$ (Table 1).

Thirty five patients (58\%) had chronic rhinosinusitis with polyps (CRSWP), 16 (27\%) were diagnosed as antrochoanal polyp, 7 (12\%) had allergic fungal sinusitis (AFS) while $2(3 \%)$ had odontogenic cysts.

The antero-inferior wall of maxillary sinus of all patients $(100 \%)$ in group (A) was visible using $0^{\circ}$ or $30^{\circ}$ endoscope. However, in 10 patients $(33 \%)$ accessibility was difficult. In group (B), the antero-inferior wall of maxillary sinus of 20 patients $(67 \%)$ was invisible and inaccessible with $0^{\circ}$ and $30^{\circ}$ endoscopes. Although $45^{\circ}$ and $70^{\circ}$ endoscopes improved visualization of the anteroinferior wall, accessibility remained limited. Comparison of accessibility between both groups showed statistically significant $P$ value $(0.004)$ (Table 2).

Intraoperative residual was detected after performing the IMF in 12 patients $(40 \%)$ at antero-inferior maxillary compartment (4 cases of antrochoanal polyps, 7 cases of CRSWP and 1 case of AFS).

Twenty eight patients $(93.3 \%)$ showed healed inferior meatal flap (Figure 2). However, 2 patients $(6.7 \%)$ showed postoperative patent inferior meatal window. 
The baseline Lund Mackay score comparison between both groups was statistically insignificant $(P=0.3)$. Similarly, postoperative 1 year comparison showed statistically insignificant $P$ value (0.6) (Table 3).

In group (A), recurrent pathology was detected at 1 year follow up in 4 cases $(13.3 \%)$ : 3 cases of CRSWP and 1 case of antrochoanal polyp. While in group (B), only 2 cases $(6.7 \%)$ had recurrence: 1 case of allergic fungal sinusitis and 1 case of CRSWP. The change was statistically insignificant with a $P$ value $(0.7)$.

No cases experienced postoperative synechiae at 2 weeks, while 3 cases $(10 \%)$ in group (A) and 2 cases $(6.7 \%)$ in group (B) experienced postoperative synechiae at $8^{\text {th }}$ week ) $\left.P<0.005\right)$.

No major complications were detected in both groups; 2 patients experienced mild epistaxis in group (A) while in group (B) 1 case experienced epistaxis, $P<0.005$ ).

Table 1: Age and sex distribution of the study's population sample

\begin{tabular}{|c|c|c|c|c|c|}
\hline \multicolumn{6}{|c|}{ Groups } \\
\hline & \multicolumn{2}{|c|}{ Group A } & \multicolumn{2}{|c|}{ Group B } & \multirow[t]{2}{*}{$P$ value } \\
\hline Age/years & Mean & $\mathrm{SD}^{*}$ & Mean & $\mathrm{SD}^{*}$ & \\
\hline \multirow{3}{*}{ Sex } & 29 & 12 & 31 & 11 & 0.6 \\
\hline & Males & Females & Males & Females & \\
\hline & 17 & 13 & 15 & 15 & 0.6 \\
\hline
\end{tabular}

* SD: Standard Deviation.

Table 2: Accessibility to the antero-inferior part of the maxillary sinus

\section{Accessibility}

\begin{tabular}{cccccc} 
& \multicolumn{2}{c}{ Group A } & Group B & P value \\
\hline & Count & $\%$ & Count & $\%$ & \\
& & & & & \\
Accessible & 20 & 66.7 & 10 & 33.3 & \\
Inaccessible & 10 & 33.3 & 20 & 66.7 & \\
\hline
\end{tabular}

Table 3: Lund Mackay score comparison between both groups

\begin{tabular}{cccccc}
\hline \multicolumn{6}{c}{ Lund Mackay score* } \\
Group A & \multicolumn{2}{c}{ Group B } & P value \\
\hline Preoperative & Count & $\%$ & Count & $\%$ & \\
2 & 12 & 40 & 16 & 53.3 & 0.3 \\
3 & 18 & 60 & 14 & 46.7 & \\
$\begin{array}{c}1 \text { year } \\
\text { follow up }\end{array}$ & Count & $\%$ & Count & $\%$ & \\
0 & 9 & 30 & 13 & 43.3 & \\
1 & 1 & 3.3 & 2 & 6.7 & 0.6 \\
2 & 2 & 6.7 & 1 & 3.3 & \\
3 & 18 & 60 & 14 & 46.7 & \\
\hline
\end{tabular}

* Scores of maxillary sinus and ostiomeatal complex only were noted.

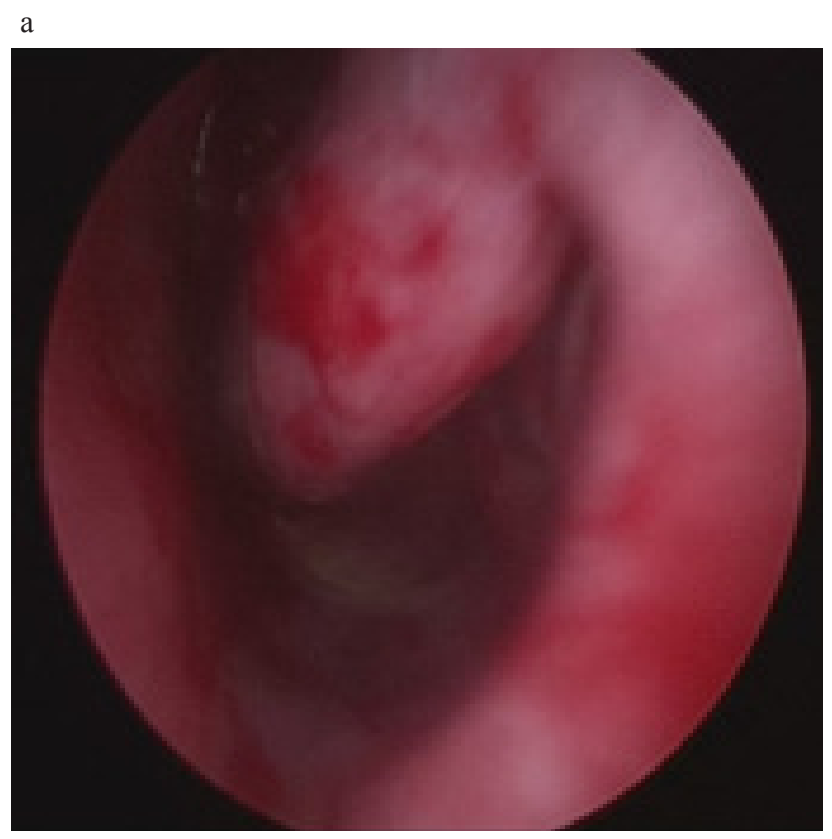

$\mathrm{b}$

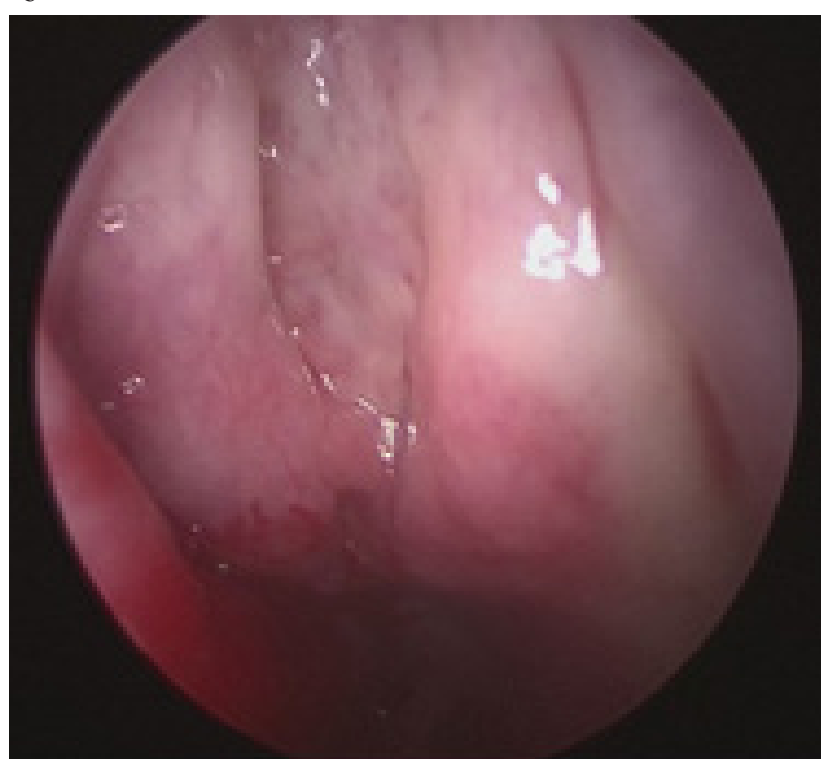




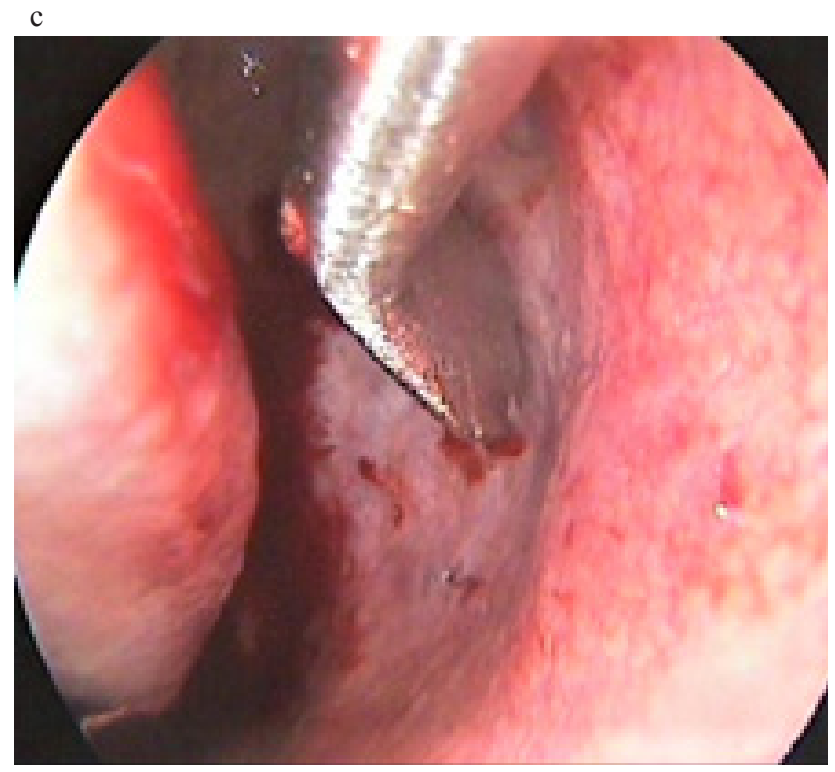

d

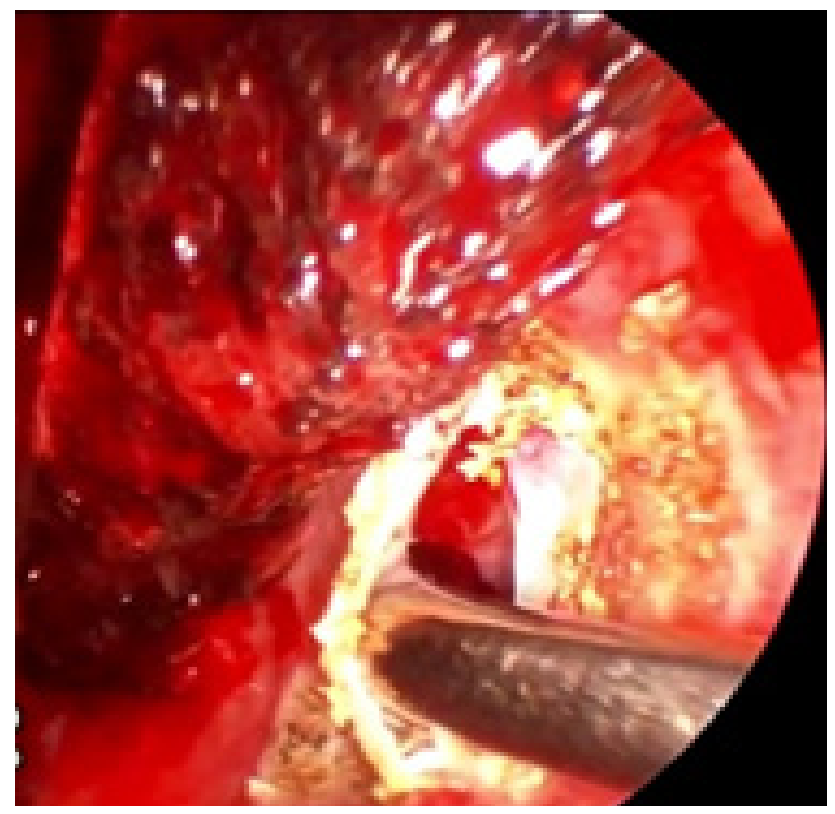

e

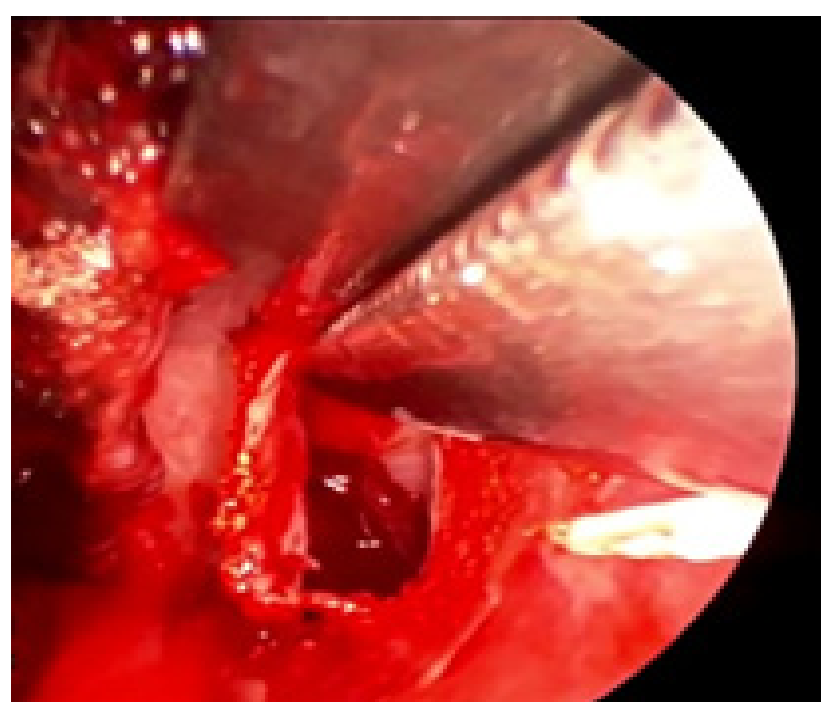

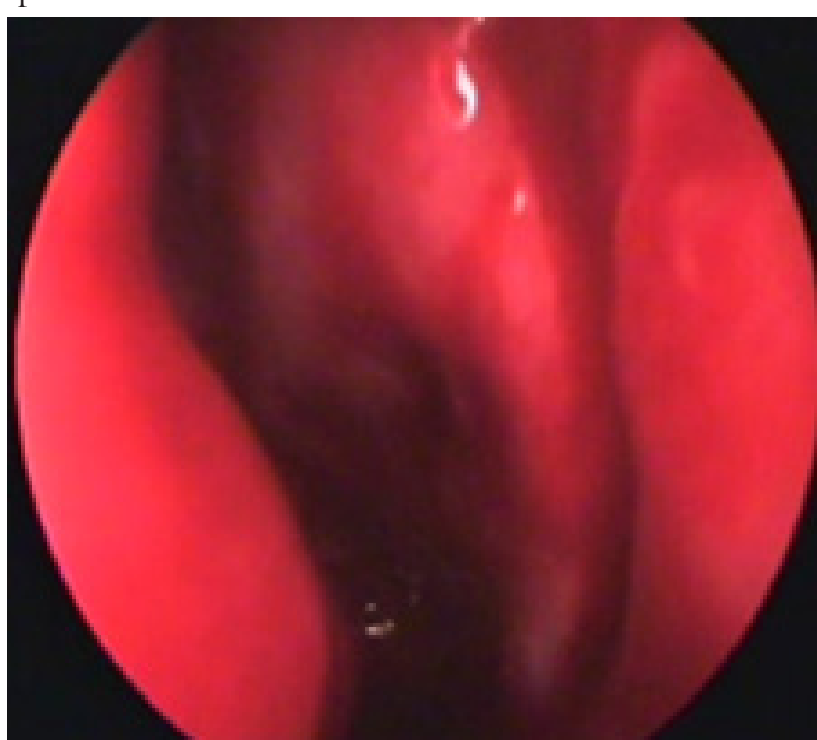

Fig. 1: Inferior meatal flap procedure on the left side; (a) Medialization of inferior turbinate, (b) Identification of Hasner's valve (asterisk), (c) Incision done anterior and inferior to Hasner's valve, (d) Elevation of the osteo-mucosal flap, (e) Division of the superior margin of the flap, (f) Lateralization of inferior turbinate after removal of the pathology.

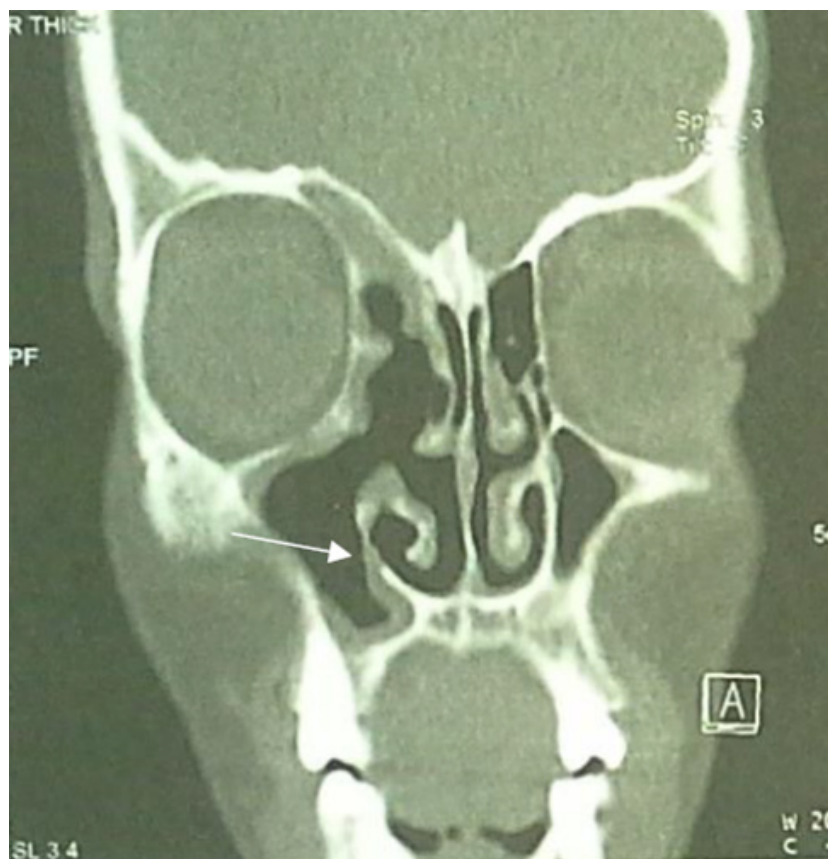

Fig. 2: One year follow up computed tomography for a case of chronic rhinosinusitis with polyps (group A), note the site of healed flap (white arrow). 


\section{DISCUSSION}

The medial and posterior parts of maxillary sinus are readily accessible. However, a lesion involving the anterior and inferior parts of the maxillary sinus is not easily reached via a trans-nasal route ${ }^{[8]}$.

In this study, addition of IMF to MMA approach had better visualization and accessibility to the antero-inferior wall of maxillary sinus being visible using $0^{\circ}$ or $30^{\circ}$ endoscopes in all patients $(100 \%)$ of group (A), nevertheless, in 10 patients $(33.3 \%)$ accessibility was hindered due to difficulty of handling instruments with the endoscope through the flap, necessitating the introduction of $45^{\circ}$ endoscope through MMA and angled instruments through IMF. On the other hand, in group (B), the antero-inferior wall of maxillary sinus of 20 patients $(66.7 \%)$ was invisible and inaccessible with $0^{\circ}$ and $30^{\circ}$ endoscopes, necessitating the use of $45^{\circ}$ or $70^{\circ}$ endoscopes, through which good visualization didn't merely mean the ability of complete lesion removal. Comparison between both groups' accessibility showed statistically significant $\mathrm{P}$ value (0.004). These results were in agreement with Michel et al., 2018 ${ }^{[9]}$ study, in which they reported good visual control of the anterior recess of the maxillary sinus using 30 degree endoscope in most cases with extended inferior meatal antrostomy.

Albu et al. ${ }^{[10]}$ in 2011 analyzed 72 patients with severely diseased maxillary sinus diseases and nasal polyps. 34 patients managed through simultaneous inferior and middle meatal antrostomies while 38 patients underwent MMA only. They affirmed the usefulness of the combined method for removing severe mucosal disease within the maxillary sinus that cannot be reached through the MMA.

The absence of wide visualization and proper instrumentation makes exploration of antero-inferior maxillary area problematic and surgery becomes difficult to perform where pathologies may remain hidden to the endoscopic surgeon ${ }^{[11],[12]}$.

Similarly in this study, despite careful removal of the pathology through MMA, residual was detected after performing the IMF in 12 patients (40\%) at antero-inferior maxillary compartment (4 cases of antrochoanal polyps, 7 cases of CRSWP and 1 case of AFS). However, after proper management of the residual, 1 year follow up Lund Mackay score comparison, did not show any statistical significance $(P=0.6)$, with recurrence of the pathology in 4 cases $(13.3 \%)$ of group A (3 cases CRSWP, 1 antrochoanal polyp), while in group (B), only 2 cases $(6.7 \%)$ had recurrence (1 allergic fungal sinusitis, 1 CRSWP). Recurrence comparison was statistically insignificant with a $P$ value $(0.7)$.
Tampering of normal mucociliary clearance by the inferior meatal antrostomy was the main concern of many authors despite the absence of consensus about $\mathrm{it}^{\mathrm{t}^{10]}}$. In this study, most of the cases $(93.3 \%)$ subjected to the IMF had complete healing, with no patent window.

Al Ayadi et al., 2015 demonstrated that without the prelacrimal recess approach (PLRA) $45 \%$ of the cases will have remnants missed in hidden areas of the maxillary sinus. In this current study, the inferior meatal flap approach (group A) could detect and clear residual pathologies in antero-inferior wall in 12 patients $(40 \%)$ even after middle meatal antrostomy, of them CRSWP, antrochoanal polyp and odontogenic cysts $^{[13]}$. Compared with endoscopic PLRA, the advantage of endoscopic inferior meatal window with a mucosal flap is less damage of the nasal lateral wall.

\section{CONCLUSION}

The inferior meatal window with mucosal flap is helpful approach to maxillary sinus hidden areas when added to standard middle meatal antrostomy. There is no patent inferior meatal window after complete healing. It is best suited for complete removal of small hidden lesions (e.g. foreign bodies, fungal ball and odontogenic cysts) which could be hardly handled through middle meatal antorstomy. However, no additional benefit was noted in decreasing the incidence of polyps' recurrence. Larger sample size is recommended for future studies.

\section{ETHICAL}

All procedures performed in this study were in accordance with the ethical standards of the institutional and / or national research committee and with the 1964 Helsinki declaration and its later amendments or comparable ethical standards.

Informed consent was obtained from all individual participants included in the study.

\section{CONFLICT OF INTEREST}

There are no conflicts of interest.

\section{REFERENCES}

1. Sathananthar S, Nagaonkar S, Paleri V, Le T, Robinson $\mathrm{S} \&$ Wormald PJ. Canine fossa puncture and clearance of the maxillary sinus for the severely diseased maxillary sinus. The Laryngoscope 2005;115(6): 1026-1029. 
2. Kaluskar S and Ohinishi T. Endoscopic sinus surgery: a practical approach. London:Springer; 1997.

3. Morgenstern L. Harold Hopkins (1918-1995): Let There Be Light Surgical Innovation 2004;11: 4291-4292.

4. Kennedy DL, Zinreich AJ, Kuhn F, Shaalan H, Naclerio R, \& Loch E. Endoscopic middle meatal antrostomy: theory, technique, and patency. The Laryngoscope 1987; 97 (S43):1-9.

5. Hosemann W, Scotti O \& Bentzien S. Evaluation of telescopes and forceps for endoscopic transnasal surgery on the maxillary sinus. American journal of rhinology 2003;17 (5):311-316.

6. Moeller CW, Stankiewicz JA. Endoscopic inferior meatal antrostomy. Oper Tech Otolaryngol 2010; 21(3):156-159.

7. Karpischenko S. Minimally invasive maxillary sinus approach. GMS Curr Posters Otorhinolaryngol Head Neck Surg 2015; 11:Doc234.

8. Lesser TH, Suryanarayanan R. Septotomy: a useful approach to the anterior maxillary sinus. The Journal of Laryngology \& Otology 2010;124(1):77-79.
9. Michel J, Radulesco T, Penicaud M \& Dessi P. Extended inferior antrostomy for maxillary sinus surgery. Clinical Otolaryngology 2018;43(2):786-788.

10. Albu S, Gocea A \& Necula S. Simultaneous inferior and middle meatus antrostomies in the treatment of the severely diseased maxillary sinus. American journal of rhinology \& allergy 2011;25(2):e80-e85.

11. Trimarchi M, Tomazic PV, Bertazzoni G, Rathburn A, Bussi M,\&StammbergerH. Video endoscopic oro-nasal visualisation of the anterior wall of maxillary sinus: a new technique. ActaOtorhinolaryngologicaItalica 2014;34(4):259.

12. Ramakrishnan VR, Suh JD \& Chiu AG. Septal dislocation for endoscopic access of the anterolateral maxillary sinus and infratemporal fossa. Am J Rhinol Allergy 2011;25:128-130.

13. Al Ayadi MA, Raafat SA, Ateya KA, Gharib FM, \& Al Murtada AM. (2015). The role of intranasal prelacrimal recess approach in complete removal of anterior maxillary sinus lesions. The Egyptian Journal of Otolaryngology, 31(4), 213. 